\title{
Rainer Döbert
}

\section{Rationalisierungseffekte durch Diskurse Beobachtungen aus einer Technikfolgenabschätzung}

\begin{abstract}
With steadily accumulating knowledge and increasing differentiation of access to knowledge democracies face the troublesome problem of technocracy. A solution was sought in widened participation without giving up the claim that ,rationality' would have a better chance of being realized. New ,constructivist' theories renounce this claim on the basis of equally valid, rationalities'. This paper tries to refute this view by specifying the concept of rationality and by analysing discourse mechanisms furthering rationality. This is done by reconstructing some lines of argumentation of a technology assessment of transgenic herbicide-resistant crops. Conclusions arise which are difficult to reject because there is a binding form of rationality at work. But rational argumentation does not guarantee consensus in politicized debates.
\end{abstract}

\section{Vorbemerkung und Übersicht}

Daß Erkenntnis übernommen wird, ist schon von Haus aus alles andere als selbstverständlich, da Selektionsprozesse involviert sind, die sich auch sozial auswirken; die Übernahme ist um so weniger selbstverständlich, als die soziokulturelle Evolution auch dazu geführt hat, daß die entsprechenden Selektionen nicht mehr von jedermann vollzogen werden können (Expertokratie) mit dem Ergebnis, daß sie zunehmend unter den Verdacht geraten sind, nicht optimal zu verlaufen. Davon handelt Abschnitt 1.1 dieses Artikels. Während die klassische Technokratiekritik davon ausgegangen ist, daß die genannten Selektionsprozesse verbessert werden können, sind in jüngster Zeit Deutungen aufgekommen, die eine Selektion von vornherein als deplaziert erscheinen lassen: Nicht bessere und schlechtere Deutungen von Technik/technischer Entwicklung konkurrieren, sondern heterogene Rationalitäten (Kosmologien), zwischen denen ,rational' selbst nicht mehr entschieden werden kann. Statt Selektion also Pluralismus! Eine solche Theorie wird in 1.2 vorgestellt. In Abschnitt 1.3 wird dann geprüft, welche verfahrenstheoretischen Implikationen sich aus den unterschiedlichen Relativierungen von Technikpolitik ergeben. Teil 2. unterwirft die konkurrierenden Deutungen dann einem empirischen Test, wozu Argumentationsgänge aus einer Technikfolgenabschätzung (TA) 
herangezogen werden. Ziel ist es zu zeigen, daß die Konflikte um die entsprechende Technik sich Rationalitätsdefiziten verdanken, die im Rahmen einer Rationalität bearbeitet werden können und die durch ,Diskursleistungen' zurechtgerückt werden. Im einzelnen wird zunächst der ,Rationalitätsbegriff ‘ eingeführt und so weit präzisiert, daß er überhaupt für empirische Analysen tauglich wird (2.1). 2.2 geht dann auf Diskursleistungen bei Wertfragen (2.2a) und Tatsachenbehauptungen (2.2b) ein. An dem, was durch Diskurse zurechtgerückt wird, wird deutlich, daß Technikkonflikte jedenfalls nicht durchgängig durch Wert- /Weltbilddivergenzen konditioniert werden. Wenn sich so am Ende die Überlegenheit einer Rationalität abzeichnet, bleibt zu fragen, ob die sich abzeichnenden Schlußfolgerungen dann auch mit Konsens/Akzeptanz rechnen dürfen. Die perlokutionäre Seite von Sprechakten in politisierten Debatten läßt dies eher unwahrscheinlich erscheinen (2.2c). Die Funktion von Diskursen mit umfassender Partizipation für demokratische Institutionen erübrigt sich damit nicht.

\section{Die Ambivalenzen von Erkenntnis und Rationalität: Elitarismus - Pluralismus - (Macht-)Diskurse \\ 1.1 Elitarismus}

Erkenntnis ist immanent elitär. Denn - und das gilt schon auf der Ebene des bloßen Wahrnehmens - wo Erkenntnis generiert wird, finden immer auch $\mathrm{Se}$ lektionsprozesse statt: Zwischen Wahrnehmungs- und Interpretationsmöglichkeiten wird entschieden in dem Sinne, daß eine (oder einige wenige) der möglichen Deutungen als ,zutreffend', ,wahr' ausgezeichnet wird und sich auf Kosten der verworfenen vorläufig durchsetzt. Da Erkenntnisse nicht im luftleeren Raum des platonischen Ideenhimmels existieren, sondern auf Erkenntnisträger, ,Subjekte', angewiesen sind, hat die genannte sachliche Selektion immer auch soziale Konsequenzen: Man wird ,ins Unrecht gesetzt', gilt als ,irrend', ,ahnungslos' etc., das heißt man wird mit Qualifikationen bedacht, die leicht endgültig disqualifizieren. Wer zu oft irrt, auf den hört schließlich niemand mehr. Nun ist dieser immanente, an die Selektivität von Erkenntnis gebundene Elitarismus für die einzelnen im Alltag ohne weiteres verkraftbar, da die Handelnden mit dem Erwerb triftiger Erkenntnis zum einen auch ihre durchschnittlichen, langfristigen Chancen erfolgreichen Handelns verbessern. Die Korrektur eines Irrtums liegt insoweit auch im Interesse des Handelnden, und nicht zuletzt deshalb wird ,Wahrheit' von uns auch als ,Wert' erfahren. So jedenfalls stellt die Situation sich dar, solange man etwa in der rationalistisch halbierten, nämlich der perlokutionären Akte beraubten Sprechakttheorie etwa von Apel/Habermas verbleibt (siehe unten).

Zum anderen gehen wir im Alltag wie selbstverständlich davon aus, daß es 
keine systematischen Ungleichheiten in der Verteilung von Irrtümern/Einsichten gibt. Im Prinzip kann jeder die richtige Selektion vollziehen und niemand ist sozusagen strukturell in der Rolle des Irrenden. Habermas/Apel's Universalpragmatik ist ein Versuch, diesen Egalitarismus der Wahrheitsfähigkeit in der pragmatischen Dimension der Sprache einzufangen.

An der Realität der modernen Gesellschaft gehen diese Präsuppositionen inzwischen über weite Strecken vorbei. Schon Simmel hat in seinen medientheoretischen Überlegungen sehr nachdrücklich darauf hingewiesen, daß sich hinter dem „kommunistischen Charakter" des Intellekts (allgemeine Mitteilbarkeit, Zugang für jedermann; Simmel 1900,603) „die unangreifbarste, weil ungreifbarste Aristokratie“, ein „Unterschied zwischen Hoch und Niedrig (verbirgt), der nicht wie ein ökonomisch- sozialer durch Dekret oder eine Revolution auszulöschen ist, und auch nicht durch den guten Willen der Betreffenden“(606f.). Der Grund für diesen ,aristokratischen“ Effekt des „kommunistischen" Mediums "Intellekt" liegt darin, daß Erkenntnisse in langwierigen und mühsamen Prozessen angeeignet werden müssen. Eine Million Geldeinheiten läßt sich in Sekundenschnelle aneignen, eine Million Sinneinheiten eben nicht. Da die soziokulturelle Evolution auch zu einer ungeheuren Massierung der Erkenntnisbestände und zugleich zu deren Ausdifferenzierung - das heißt sozial: zu ihrer Unzugänglichkeit jeweils für Teile der Bevölkerung - geführt hat, hat die Differenzierung zwischen ,Hoch und Niedrig' ein historisch unerreichtes Niveau erreicht, das man nach Simmel auch „durch den guten Willen der Betreffenden" nicht zurückschrauben kann.

Nehmen wir zu all dem hinzu, daß die Evolution auch zu einer ungeheuren Beschleunigung des Tempos der Erkenntnisproduktion geführt hat (vgl. Luhmann 1984), dann ist völlig klar, daß der einzelne objektiv nur noch in Ausnahmefällen in der Lage sein wird, die für Erkenntnis konstitutiven Selektionsprozesse auf der Basis des gegebenen Erkenntnisstandes vorzunehmen. Seine subjektive Einschätzung mag und wird - das postmaterialistische Wertsystem (Inglehart 1989) ist schließlich auch als Narzißmus (Lasch 1982) beschrieben worden - in vielen Fällen zu einem anderen Ergebnis kommen; das Problem ist jedoch prinzipiell auf der individuellen Ebene unlösbar. Faktisch haben sich auch andere Lösungen eingespielt, die auf Delegation von Entscheidungskompetenz aufbauen. $\mathrm{Zu}$ nennen sind Technokratie und repräsentative Demokratie, die im Zusammenspiel ihrer Institutionen die Sachrationalität verwirklicht, welche man auf der Ebene der Wählerschaft grundsätzlich nicht erwarten darf (vgl. Sartori 1992).

Nun steht diese quasi-apriorische Legitimation von Technokratie und äußerst eingeschränkten demokratischen Mitgestaltungsmöglichkeiten empirisch ersichtlich auf sehr schwachen Füßen. Die andere Lösungsmöglichkeit, von der ich sagte, sie habe sich ,eingespielt', hat sich gegenwärtig anscheinend eher ,ausgespielt'. Die entsprechenden Phänomene sind hinreichend bekannt und 
müssen hier nicht mehr erwähnt werden. Mit der postmaterialistischen Kehre sind auch Partizipationsforderungen intensiviert und zunehmend durchgesetzt worden (Bürgerinitiativen etc., vgl. Rucht 1996). Die Technikfolgenabschätzung von der hier berichtet wird, ist ja auch nur ein Beispiel für Prozeduralisierungstendenzen (Teubner 1989), Deregulierungen und neokorporatistische Arrangements, mittels derer der Staat zugunsten der Selbstorganisation von Gesellschaft (vgl. Weidner 1996) zurückgedrängt werden soll. Das Vertrauen in die Technokratie ist eben in starkem Umfang geschwunden, weil der Bürger/die Bürgerin sich an der Umweltkrise, den Risiken der Kernkraft etc., ja an der Widersprüchlichkeit der Expertenaussagen selbst ohne Schwierigkeiten vergegenwärtigen kann, daß die Delegation von Rationalität nicht unbedingt Garant von Rationalität ist - auf jeden Fall aber nicht Garant dessen, was er/sie unter einem guten Leben verstehen mag.

Daher überrascht es nicht, daß der Strom der expertokratiekritischen Literatur in den letzten Jahren sichtbar angeschwollen ist (vgl. sehr früh: Habermas 1968; neuere Übersichten bei Hawkesworth 1988; Fischer 1990). Die Hauptargumente gegen das expertokratische Denken sind: (1) Mit der strikten Trennung zwischen deskriptiven und präskriptiven, wissenschaftlichen und politischen (Wert-)Fragen wird der Prozeß der rationalen Deliberation auf Sekundäres, nämlich die Mittelwahl, beschränkt, und die für die Wahl einer Lebensform wirklich entscheidenden Wertfragen werden fast gänzlich der Irrationalität überantwortet. (2) Die Experten sind nur scheinbar wertneutral, weil sie in Wirklichkeit mit ihren Entscheidungen auch latente Wertungen transportieren. (3) Die Usurpation der politischen Entscheidungen durch die Experten führt zu einer Entpolitisierung der Bevölkerung, die das demokratische System nur noch weiter aushöhlen kann.

Diese Argumente zielen nicht nur auf Technikpolitik ab, aber sie sind in jüngster Zeit stark auf diesen Politikbereich fokussiert worden; dies in erster Linie natürlich, weil die Umweltkrise eine Fehlentwicklung von Technik anzeigt; dies aber auch, weil die Technikentwicklung weitgehend im ökonomischen System, jenseits demokratischer Kontrollen, vorangetrieben wird. Technikentwicklung ist, gesellschaftspolitisch, Evolution, nicht rationale Gestaltung. Und genau diesen Zustand will man nicht länger hinnehmen; technische Anlagen, technische Innovationen und ganze Technikfelder - Stichwort: Gentechnik - stoßen daher auf einiges an Widerstand, ja, man kann sagen, daß die Konflikte im Bereich der Technikentwicklung sich durch besondere Hartnäckigkeit und Unversöhnlichkeit auszeichnen.

Abhilfe versprechen sich sowohl Herrschende wie Beherrschte von erweiterten Partizipationsmöglichkeiten (Zulassung bei Anhörungen, Mediationsverfahren, Konsensuskonferenzen, Technikfolgenabschätzungen etc.). Dadurch wird auf jeden Fall Einfluß auf Selektionsprozesse von der etablierten Technokratie auf größere Kreise verlagert. Das ist die herrschaftssoziologische Sei- 
te. Damit ist über die Sachrationalität der dann generierten Entscheidungen nichts gesagt. Um die Implikationen der sozialen Dimension (Beteiligungsrechte von wem?) für die Sachdimension geht es aber zentral in diesem Beitrag. Die ,klassische' Technokratie-Kritik ist in der Regel davon ausgegangen; daß durch erweiterte Partizipation auch sachlich angemessenere Selektionen - von konkurrierenden Deutungen, von konkurrierenden normativen Vorstellungen - zustande kommen. Die Technikkonflikte hätten ihre Ursache demnach (auch) in Rationalitätsbeschränkungen, denen man wenigstens zum Teil durch Aufnahme von Diskursen entgegenwirken könnte. Die Rationalitätsbeschränkungen können sich aus der Vernachlässigung von Wertgesichtspunkten, aus Erkenntnisdefiziten/Ungewißheit oder einseitigen Interessenlagen ergeben. Vernachlässigte Werte können in Kompromißkonstruktionen Berücksichtigung finden, Unsicherheit über die Auswirkungen oder die Sicherheit von Techniken lassen sich durch vermehrte Forschung und Sicherheitsauflagen teilweise abbauen, interessenbedingten Einseitigkeiten bei der Informationsverarbeitung läßt sich durch die Präsenz ,anders' interessierter Akteure entgegenarbeiten etc. (Naess 1989; Beck 1986; Keck 1993).

\subsection{Pluralismus}

Die konstruktivistische Wende in den Humanwissenschaften hat dann aber auch Deutungen dieser Kontroversen hervorgebracht, angesichts derer eine rationale Entscheidung zwischen wahr/falsch, angemessen/unangemessen eigentlich überhaupt nicht mehr gedacht werden kann. Jedenfalls müssen diejenigen, deren Deutung zurückgewiesen wird, nicht mehr davon ausgehen, daß sie ,falsch' denken; vielmehr können sie in der beruhigenden Gewißheit, daß sie einfach ,anders' denken, an all ihren Ideen/Urteilen festhalten. Die elitären Implikationen von Sinn/Intellekt sind damit - sowohl in der basalen wie in der evolutionär verschärften (Simmel) - Version weitgehend beseitigt. Es gibt nicht Rationalität, sondern ,Rationalitäten'. Erkennbar handelt es sich um eine Variante des für moderne Gesellschaften typischen Pluralismus, der nun allerdings Bereiche erobert, die bislang auf Selektion angelegt waren.

Im einzelnen kann diese Sichtweise der Technik- und Umweltkonflikte sehr unterschiedlich ansetzen (hermeneutischer Zirkel, unterschiedliche framings, autopoetische Geschlossenheit; vgl. Hawkesworth 1988; Jungermann/Slovic 1993; Luhmann 1986). Im deutschsprachigen Raum hat wohl die Systemtheorie Luhmanns mit ihrer autopoetischen Wendung den größten Einfluß gehabt. Sehr stilisiert zusammengefaßt läuft sie auf die These hinaus, daß die ausdifferenzierten gesellschaftlichen Teilsysteme füreinander ,blind' sein müssen, weil sie je andere Codes (Wahrheit, Geld, Macht etc.) verwenden und mit divergierenden Wahrnehmungsschemata operieren. So prallt z. B. die Ökologie an der Ökonomie einfach ab (Luhmann 1986; Japp/Krohn 1996). Hier soll jedoch 
nicht die Theorie Luhmanns, sondern die cultural theory der Gruppe von M. Douglas (Douglas 1992; Thompson/EllisWildavsky 1990) auf den Prüfstand gestellt werden. Denn die Konflikte um Techniken werden überwiegend zwischen Vertretern von Teilsystemen (Wirtschaft, Wissenschaft, Politik) auf der einen Seite und Mitgliedern von Organisationen, die keinem spezifischen Teilsystem zugerechnet werden können (Umweltbewegungen) auf der anderen Seite ausgefochten. Die ,Logik' des Denkens der Umweltbewegungen wird von Douglas genauer analysiert als dies bei Luhmann der Fall ist, und dies genau mit dem Ziel, eine theoretische Erklärung für die Hartnäckigkeit und argumentative Starrheit umweltpolitischer Konflikte zu liefern. Ihre Theorie soll kurz vorgestellt werden, um sie dann im Kontext der konkurrierenden Deutungen von ,Unversöhnlichkeit' auf ihre verfahrenslogischen Implikationen hin zu untersuchen. Damit wird dann die Fragestellung dieses Artikels hinreichend präzisiert sein.

Douglas versucht nach klassischer soziologischer Manier, Deutungsmuster aus sozialstrukturellen Konfigurationen (mit den daran geknüpften typischen Erfahrungen) umfassende ,Kosmologien' abzuleiten, die jeweils ihre eigene Rationalität verkörpern. Dabei faßt sie die Sozialstruktur in zwei Dimensionen, nämlich der Zahl der einem Individuum vorgegebenen, verbindlichen Kategorisierungen/Vorschriften (grid) und dem Grad der Gruppensolidarität (group). Die Deutungsmuster beschreibt sie in den Dimensionen von Wertsystem, Naturkonzept und Risikoerwartung. Wenn grid/group jeweils hoch/niedrig sein können, ergeben sich vier Weltbilder. Bei hohem grid und group (Bürokratie, Mitglieder einer Kaste) verbindet sich eine Schätzung von Hierarchie/Expertentum (Wert) mit dem Bild einer bedingt stabilen Natur, in die man mit kalkuliertem Risiko eingreifen kann.

Von wirklich entscheidender Bedeutung ist, daß zu diesem Weltbild der Hierarchie auch die moderne Wissenschaft gehört, die damit jeden Sonderstatus verliert: Sie ist eine ,Kosmologie' neben anderen - ohne Anspruch auf überlegene Verfahren der Sicherung von Geltung. Bei niedrigem grid und hoher Gruppensolidarität (egalitäre Gemeinschaft) verbindet sich der Wert des Egalitarismus mit der Schematisierung einer extrem labilen Natur, die weiter auszuplündern ein enormes Risiko bedeuten würde. Wo grid und group niedrig sind (freies Unternehmertum) setzt man auf den Wert Individualismus und geht von einer äußerst stabilen Natur aus - schadlos verschwindet der Konkurrent vom Markt, schadlos wird ein Biotop entfernt - in die recht risikolos eingegriffen werden kann. Und schließlich findet man bei hoher Regelungsdichte (grid) und fehlender Gruppensolidarität (anomischer Hilfsarbeiter), einen Fatalismus, bei dem auch der Lauf der Natur erratisch ist.

Die genannten ,Kosmologien' sollen sich wechselseitig ausschließen und unterschiedliche ,Logiken“ definieren: „Because the myths that define the four engaged ways of life are mutually contradictory, they each define as rational 
behavior what the others define as irrational" (Thompson et al. 1990, 31). Und das ist genau der Punkt: Wenn dem einen als irrational gilt, was dem anderen Inbegriff von Rationalität ist, dann sind beide Seiten argumentativ füreinander unerreichbar. Das ist gut so, zumindest für ,Laien', denn diese denken/argumentieren nicht schlechter als Experten, sondern ,anders'.

\subsection{Diskurse und Zufalls- oder Machtspiele}

Diese Zuspitzung hat nun weitreichende Implikationen. Denn von den sozialwissenschaftlichen Deutungen hängt auch ab, was Diskurse wie z. B. Technikfolgenabschätzungen für Gesellschaften überhaupt leisten können. Offiziell sollen sie rationale, $\mathrm{d}$. h. begründete Urteile über Technik ermöglichen und dadurch einen Konsens stiften (Integrationsmedium: Argumentation). Im Unterschied zu den älteren Deutungen schließen die neueren diese Lesart der offiziellen Funktion von TAen jedoch fast grundsätzlich aus. Anhand einer kleinen Grafik gebe ich zunächst einen Überblick.

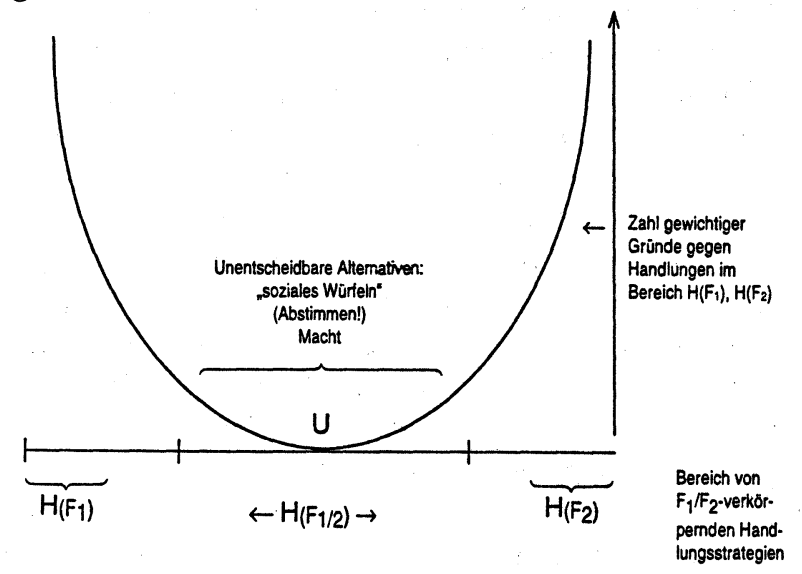

Deutungen von Technikkonflikten:

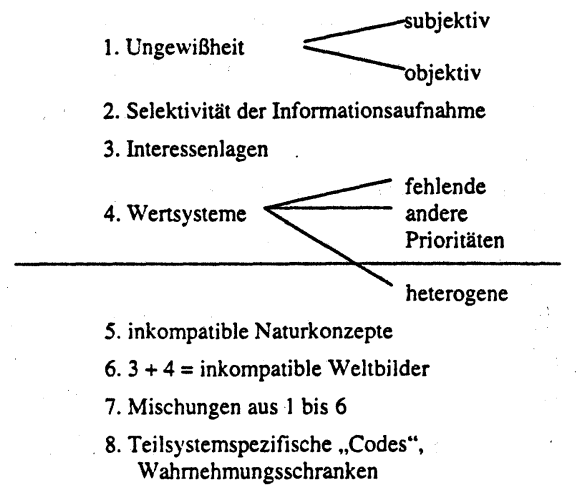


Ich schließe dabei an Rawls' quasi reine Verfahren an, die auf der Beobachtung beruhen, daß bei den meisten gesellschaftspolitischen Kontroversen gewisse extreme Handlungsoptionen mit guten Gründen ausgeschlossen werden können, daß danach aber meist ein ganzer Bereich von Strategien verbleibt, über die argumentativ nicht entschieden werden kann (Rawls 1971, 201, 261f.). Welche dieser Strategien dann auch immer gewählt wird, sie verdankt ihre Selektion nicht sachlicher Angemessenheit (rational). An der Konkurrenz von Funktionen, z. B. Ökonomie und Ökologie, kann man sich den Sachverhalt verdeutlichen. Wir können weder nur Ökologie noch nur Ökonomie machen. Diese monofunktionalen Optionen lassen sich argumentativ/rational ausschalten, weil die vernachlässigte Funktion starke Gründe liefert. Die Bereiche $H\left(F_{1}\right)$ und $H\left(F_{2}\right)$, die Handlungsoptionen mit Primat der Funktionen $F_{1}$ und $F_{2}$ enthalten, lassen sich also diskursiv ,abschneiden'. Im Bereich $U$ verbleiben dann Kompromißstrategien $\left(H F_{1} / F_{2}\right)$, zwischen denen mit Gründen nicht entschieden werden kann. Der Entscheidungsmechanismus kann also nichts mit Rationalität zu tun haben, sondern muß als Macht- oder Zufallsspiel interpretiert werden. Die Frage ist dann natürlich, wie unterlegene Parteien mit Ergebnissen von Macht- oder Zufallsspielen umgehen werden. Sicherlich anders als mit Ergebnissen überzeugender Argumentation!

Somit hängt einiges an der Breite der Bereiche $U$ und $H$ und an ihrer Unterscheidbarkeit: Wenn es nur $U$ gibt, ist Argumentation von Grund auf sinnlos. Schaut man sich gängige Deutungen von sozial- und technikpolitischen Kontroversen an, zeigt sich, daß das Verhältnis von $U$ und $H$ und die Chancen, $U$ in $H_{1}$ oder $H_{2}$ zu verwandeln, höchst unterschiedlich eingeschätzt werden. Ich habe einige der relevanten Deutungen stichwortartig unter der Grafik zusammengestellt und durch einen Querstrich getrennt. Unterhalb dieser Linie ist Argumentation mit Konsenschancen fast nicht mehr denkbar. Gehen wir die Implikationen der einzelnen Faktoren kurz durch:

Die Rolle von ,Ungewißheit', die ja speziell bei der Komplexität großtechnischer Anlagen und ökologischer Systeme (Kernkraft, Chemie; vgl. Perrow 1987; Weber 1994) immer wieder dramatisiert wird, ist ambivalent. Auf der einen Seite läßt sich ,Ungewißheit' durch vermehrte Forschung, durch Poolen von Information beseitigen. Dann ist Verständigung möglich. Aber es verbleibt eine Restungewißheit, die unterschiedlich genutzt werden kann. ,Ungewißheit‘ bedeutet einerseits, daß man Fehlentwicklungen nicht antizipieren kann und daher Optionen verfolgt, die eigentlich in $H\left(F_{1}\right)$ oder $H\left(F_{2}\right)$ gehören. Man arbeitet mit einem zu breiten $U$. Das ist typisch für Douglas' Individualisten. Seit derartige Fehlentwicklungen dingfest gemacht wurden, wird Ungewißheit andererseits nur noch zur Beschränkung von $U$ benutzt: Im Unbekannten lauern Gefahren, die technische Innovationen verbieten (vgl. van den Daele 1997). Damit sind wir bei den extremen Risikostilisierungen, die für Douglas' egalitäre Gemeinschaften charakteristisch sind. Wie werden Douglas' Indivi- 
dualisten aufgrund ihrer Risikobereitschaft mit diesen Risikodramatisierungen umgehen? Die Frage läßt sich nur auf der Meta-Ebene beantworten. Wenn wir mit Douglas davon ausgehen, daß hier heterogene Weltbildelemente aufeinandertreffen, dann ist klar, daß zwischen beiden Lagern ein argumentatives Patt bestehen muß - beide Konzeptionen gehören in den Bereich von $U$. So stellt es sich jedenfalls für den Beobachter der Kontroversen dar.

Mehr Eindeutigkeit ergibt sich bei den in der Liste folgenden Faktoren. Wenn die Konflikte darauf beruhen, daß die verfügbare Information nur selektiv genutzt wurde, haben Diskurse gute Chancen. Denn schon durch die Präsenz der Opponenten ergibt sich eine neue Argumentationslage. Das Gleiche gilt für Interessenlagen. Denn zum einen setzen Interessenkonflikte eine geteilte Situationsdefinition voraus. Zum andern gibt es eine Beziehung zu selektiver Informationsverarbeitung. Niemand wird sagen, Argument $x$ oder $y$ akzeptiere ich nicht, weil es meinen Interessen nicht entspricht. Man wird es einfach ausblenden und auf dieser Basis den Anschein erwecken wollen, die eigene Option $H$ liege im Bereich $U$. Da die Opponenten jedoch am,Verdrängten' interessiert sind, werden sie dafür sorgen, daß $H$ schließlich zurückgewiesen und $U$ wieder kleiner wird. Wertdivergenzen wirken sich unterschiedlich aus, je nachdem wieweit man sie ,prinzipialisiert'. Das Beiseiteschieben eines Werts, dessen Geltung man nicht grundsätzlich bestreitet, führt zu einer Ausweitung von $U$, die sich rückgängig machen läßt. Räumt man einem Wert geringeres Gewicht ein als die Gegenseite, wird man Handlungsoptionen für vertretbar halten, die diese ablehnt. Die Einwände der anderen können nur bedingt etwas ausrichten; aber man wird auch nicht bis zu den Extrempunkten $H\left(F_{1}\right)$ und $H\left(F_{2}\right)$ gehen, da man den entsprechenden Wert selbst auch noch ,kennt'. Bei all' diesen Deutungen gibt es also noch einen Unterschied zwischen $U$ und $H\left(F_{1}\right), H\left(F_{2}\right)$; und das heißt, daß argumentativ wenigstens Teilkonsense erreichbar sind.

Wenn diese Voraussetzung entfällt, wenn also zum Beispiel Werte wie ,landwirtschaftliche Rentabilität' auf der einen Seite und ,Artenvielfalt im Agrarökosystem' sozusagen als Bewohner völlig fremder Planeten aufeinandertreffen, dann dehnt sich $U$ über das ganze Handlungsspektrum aus, da die jeweiligen Argumente des Opponenten keine Kraft haben können. Dies gilt erst recht, wenn nicht nur heterogene Werte, sondern völlig unvergleichliche, inkompatible Kosmologien aufeinandertreffen, wie es etwa Douglas et al. unterstellen. Nicht anders stellt sich die Situation dar, wenn man die relative Autonomie der gesellschaftlichen Teilsysteme zu ,Autopoese' übersteigert oder mit dem postmodernen Konstruktivismus die Perspektivität jeder vorläufigen Deutung für das letzte Wort (keine Selektionen!) hält (vgl. Wagner 1995). ,Wissenschaft' ist dann auch nur eine der Perspektiven, die gleichberechtigt mit dem Stammtischgerede konkurriert - und dann qua Macht/Abstimmen konsequenzenlos unterliegen kann. 
Nach den neueren - kosmologisch geweihten - Deutungen technikpolitischer Kontroversen sind die Chancen dafür, daß mit einem Konsens auf der Basis überzeugender Sachargumente gerechnet werden kann, mithin gleich Null. Die offizielle Hauptfunktion von partizipativen Arrangements ruht auf einem falschen Schein von Argumentationsmöglichkeiten, die die relative Rationalität/Legitimität der Ausgangspositionen letztlich nicht tangieren können. Die Teilnehmer beispielsweise von Technikfolgenabschätzungen sehen das durchaus anders - und argumentieren unverdrossen. Natürlich können sie dabei dem ,falschen Schein' aufsitzen. Ehe man sie derart in's Unrecht setzt, sollte man aber doch vielleicht auch einmal empirisch überprüfen, ob und welche Argumentationsgänge am Ende nicht doch hinreichend Kraft entfaltet haben, um Selektionen von wahr/falsch vorzubereiten. Schließlich darf auch nicht vergessen werden, daß die genannten sozialwissenschaftlichen Deutungen - unbeabsichtigt oder nicht - auch die zentrale Ressource der Umweltbewegung, nämlich gute Argumente, unterminieren.

Genau von einem solchen empirischen Test, nämlich einer Technikfolgenabschätzung von gentechnisch erzeugter Herbizidresistenz soll im folgenden soweit berichtet werden, wie es für die Zwecke dieses Artikels erforderlich ist. Die Kontroverse um die Gentechnik zeichnet sich durch die besondere Hartnäckigkeit aus, die Anlaß zu den genannten ,kosmologischen Spekulationen' gegeben hat. In ihr spielen neue Wertvorstellungen, zum Beispiel ein moralischer Respekt vor der Integrität der Genome der Arten, eine Rolle (vgl. Altner 1994), und sie berührt unser Naturverständnis. Zweifellos ist die Kontroverse um die Gentechnik in der Öffentlichkeit auch genau mit der Rhetorik belegt worden, die auf eine Konfrontation zwischen inkompatiblen Weltbildern hinweist. So ergibt sich insgesamt also ein gutes Testfeld für die Leistungsfähigkeit diskursiver Verfahren. Erzeugen sie Rationalitätsgewinne, führen sie zu einem Konsens, wenn sich klare Argumentationslagen ergeben? Diese Fragen sollen schrittweise abgearbeitet werden.

Zunächst einmal ist das bislang unexplizierte Konzept der Rationalität soweit zu klären, daß schließlich auch die empirischen Phänomene, um die es hier geht, eingeordnet werden können. Dann ist kurz auf die Dimension der Werte einzugehen. Weiterhin ist wenigstens so viel an Diskurstheorie zu erörtern, daß deutlich wird, welche Rationalisierungseffekte im einzelnen erwartet werden können. Am Beispiel einzelner Argumentationsblöcke soll dann demonstriert werden, wie Rationalisierungseffekte wirksam geworden sind. Ein Block wird sich auf ,Unsicherheit' beziehen, ein Begriff, der im Weltbild von Douglas' Egalitären eine herausragende Rolle spielt. Ein anderer Block wird Selektivität der Informationsaufnahme zum Thema haben: Soweit sich Selektivität nachweisen läßt, ist diese - nicht ein ,Weltbild` - Grund für die scheinbare Plausibilität der eigenen Vorstellungen. 


\section{Diskursive Rationalität}

\subsection{Zum Rationalitätskomplex}

Ich habe den Rationalitätsbegriff bislang unexpliziert gelassen und dabei darauf vertraut, daß der Begriff beim Leser schon in etwa mit den richtigen Assoziationen verknüpft ist. Wir müssen den Begriff nun aber etwas genauer fassen und vor allem auffächern, damit diskursive Rationalisierungsleistungen sichtbar werden. Nur wenn wir schwer abweisbare Rationalitätsgewinne an einzelnen Argumentationsounkten nachweisen können, läßt sich der Rhetorik von den gleichberechtigten Rationalitäten entgegentreten. Denn eines liegt ja auf der Hand - daß nämlich die argumentativ Unterlegenen im Zweifelsfalle das Schlupfloch ,anderes Weltbild' schon finden werden.

Soziologen denken bei „rational“ am ehesten an Webers Wert- und Zweckrationalität oder ,rational choice', Philosophen rekurrieren am ehesten auf den Begriff der ,Begründung', wenn sie Rationalität explizieren sollen: Rationales Handeln liegt vor, wenn der Handelnde für seine Handlungsoption ,Gründe', ,Argumente' angeben kann, die es erlauben, die (soziale oder nichtsoziale) Handlung (unter gegebenen Randbedingungen) zu ,empfehlen'; Begründungszwänge ergeben sich im sozialen Verkehr spätestens bei Störungen von Routinen, ja, soziale Interaktion ist der Motor von Begründung.

Der Begriff der Begründung deckt wert- und zweckrationales Handeln ab, da beide Varianten begründeten Handelns sind. Beim wertrationalen Handeln kann der Handelnde auf einen (vermeintlich) geltenden Wert, eine geltende Norm verweisen, deren Geltung die Handlung zum Ausdruck bringt („man muß anderen doch helfen, deshalb spende ich ..."). Wenn diese Norm in Frage gestellt wird, kann sie gegebenenfalls unter Rekurs auf höherrangige Normen begründet werden; so bauen sich dann die deontologischen Begründungshierarchien auf. Beim zweckrationalen Handeln wird als Handlungsgrund darauf verwiesen, daß der geplante Eingriff in die Handlungssituation (die Mittelhandlung) nach bestem Wissen ein gegebenes Handlungsziel zu erreichen erlaubt. Das ,beste Wissen' kann in die Irre gehen und in Frage gestellt werden; dann können die ,Gründe ${ }^{\natural}$ und ,Gegengründe', Evidenzen und Gegenevidenzen nach etablierten kognitiven Standards gesichtet werden, um sich der „objektiven Richtigkeitsrationalität" (Weber) des ,besten Wissens' zu vergewissern.

So ergibt sich eine Begründungshierarchie des zweckrationalen Handelns. Die Handlung kann dabei durchaus so etwas Komplexes sein, wie ,Kampf gegen die oder ,Förderung' der Gentechnik. Der ,Kampf' wird mit Risikobehauptungen begründet, die bestritten werden etc., so daß schließlich eine ganze Batterie von wissenschaftlichen Gutachten und Gegengutachten mobilisiert werden muß. All diese Ausflüge in die Höhen oder Tiefen wissenschaftlicher Rationalität werden aber nur unternommen, um Gründe für die Handlungen, „Kampf' vs. ,Förderung' zu gewinnen. ,Rational choice' und ähnliche forma- 
lisierte Modelle (Spieltheorie etwa als soziale Version) sind nur präzisierte Varianten von zweckrationalem Handeln. Meist steht mehr als eine Mittelhandlung zur Erreichung eines Ziels zur Verfügung, so daß eine Auswahl zu treffen ist. Diese Auswahl muß dann im Rahmen einer Theorie der rationalen Wahl nicht ,intuitiv', nach schwankender Einstellung vollzogen werden, sondern orientiert sich an einem Rationalitätskriterium - in diesem Fall am Kriterium Nutzen $\times$ Eintrittswahrscheinlichkeit des Erfolges. Darüber, welche Rationalitätskriterien wann einzusetzen sind, herrscht Streit. Rawls beispielsweise operiert mit dem Maximin-Kriterium (in etwa: größter erwarteter Nutzen bei ungünstigstem Verlauf; siehe Rawls 1971). In jedem Fall aber gewinnt der Handelnde bei Verwendung eines dieser Rationalitätskriterien einen zusätzlichen Handlungsgrund: Durch die Handlung wird ein Zweck erreicht, und von allen Handlungen, die dies vermöchten, wird diese eine dies ,am besten' tun. Damit ist also eine Begründungslücke geschlossen.

So viel zum Kern des Weber'schen Rationalitätskomplexes und seiner Beziehung zur philosophischen Begrifflichkeit (vgl. Döbert 1989). Ich spreche von einem ,Rationalitätskomplex', weil der Rationalität des begründeten Handelns eine Ebene vor und eine Metaebene nachgelagert sind. Die eine schafft eine Minimalvoraussetzung von Begründung/Argumentation: Ein Handlungsfeld muß überhaupt erst ,bewußt' ins Auge gefaßt, der Routine entzogen werden, um dann nach Gründen organisiert zu werden. Die Umstellung der Technikentwicklung von Evolution auf politische Gestaltung berührt genau diese Komponente des Weber'schen Rationalitätskomplexes. Die Veranstaltung von Technikfolgenabschätzungen ist also per se, unabhängig von ihren internen Dynamiken, schon ein Rationalisierungsprozeß im Weber'schen Sinne, aber einer, den wir hier in seiner Bedeutung nicht analysieren, sondern voraussetzen.

Wichtiger für unsere Zwecke ist die angesprochene Metaebene des Rationalitätskomplexes, auf der nicht Gründe für einzelne Handlungen, sondern generalisierte Gründe, die die Form/Organisation von Gründen der ersten Ebene betreffen, angesiedelt sind. Weber spricht bei Handlungsbereichen von deren „Berechenbarkeit", „maschinenmäßigem Funktionieren“; geht es um Sinnsysteme, ist von deren „Logifizierung" und „Systematisierung“ die Rede. Diese Termini betreffen nicht Gründe für einzelne Handlungen oder Argumente, sondern die Struktur ganzer Handlungsfelder und Gedankenbereiche. Diese sollen so organisiert sein, daß Handlungen/Argumentationsgänge ihr Ziel mit Sicherheit, Genauigkeit, ohne Widersprüchlichkeiten oder Störung durch unberücksichtigte Gesichtspunkte (Systematik heißt auch: Vollständigkeit) erreichen. Diese Ebene verkörpert die höchste Stufe der Rationalisierung eines Handlungsbereichs. Sie entspricht in etwa dem, was Rawls mit seinem „wohlerwogenem Urteil im reflexiven Gleichgewicht" vor Augen hatte. Und dabei handelt es sich gerade nicht um ein Kunstprodukt des philosophischen Elfenbeinturms. 
Die Teilnehmer/Teilnehmerinnen an der Technikfolgenabschätzung von herbizidresistenten Kulturpflanzen haben in einer Eingangsbefragung recht einhellig die Auffassung vertreten, daß sie ein Urteil über die HerbizidresistenzTechnik nur dann als ,begründet' anerkennen könnten, wenn wirklich alle relevanten Informationen über die Technik gesichtet und gewogen würden: Sie verlangten „sachliche Repräsentativität" (Bora/Döbert 1993). Sie verlangten gleichzeitig, daß alle relevanten Wertungen im TA-Diskurs eine Stimme finden würden (soziale Repräsentativität). Im Umkehrschluß ist damit auch klar, daß die Beteiligten eine Selektivität der Informationsaufnahme bei der Beurteilung der Technik als Rationalitätsdefizit anerkennen würden. Ziel dieses Artikels ist es zu zeigen, daß und durch welche Mechanismen ein Technikfolgenabschätzungs-Diskurs unter angemessenen Randbedingungen auf Rationalität in diesem stärksten Sinne zuläuft.

Wie hat man sich dieses Zulaufen und sein Ergebnis in etwa vorzustellen? Sachliche und soziale Repräsentativität sorgen ja zunächst einmal nur für eine Mannigfaltigkeit von Behauptungen/Gegenbehauptungen und divergierenden Bewertungen. Diese müssen selektiv verdichtet werden, ohne daß berechtigte Argumente/Gegenargumente verloren gehen. Woran erkennt man, ob eine schließlich gezogene Konklusion diesen Anforderungen genügt? Nur wenn dafür wenigstens eine Heuristik zur Verfügung steht, wird man gegebene Schlußfolgerungen als ,begründet' oder ,unbegründet' einstufen können. An dem Punkt ist eine Beobachtung Piagets über die Verarbeitung von kognitiven Störungen/Gegenargumenten hilfreich. Piaget spricht in dem Zusammenhang von "Negationen“ der Ausgangsbehauptungen, die es kognitiv zu ,internalisieren" gilt. Er konnte dabei drei Etappen unterscheiden (Piaget 1975):

1. Die Negationen (abweichende Daten/Argumente) werden verdrängt, beiseite geschoben. Das hat ersichtlich mit der mehrfach erwähnten Selektivität der Informationsverarbeitung zu tun.

2. Es kommt zu einer Differenzierung derart, daß den widersprüchlichen Daten unterschiedliche Situationen zugeordnet werden.

3. Es gelingt, ein umfassendes Konstrukt oder eine mehrdimensionale Theorie zu entwickeln, aus der sich die unterschiedlichen Situationen konsistent ableiten lassen. Das, was ursprünglich als abweichende Beobachtung oder als Gegenargument (Negationen) störte, kann nun als eine normale Variante des Geschehens behandelt werden. Eine Verdrängung der Negationen ist nicht mehr erforderlich, weil diese in dem neuen Konstrukt, so Piagets Terminologie, internalisiert sind.

Um ein Beispiel aus einem Umweltdilemma zu geben, bei dem menschliche Erholungsbedürfnisse und der Schutz eines Biotops (Badesee) konfligieren (Döbert/Gericke 1997): Eine Verleugnung findet statt, wenn man für 
den Schutz des Biotops plädiert, ohne die Erholungsbedürfnisse auch nur zu erwähnen. Als Differenzierung kann gelten: „Die Menschen sollen nur in einem Teil des Sees baden, in dem anderen die Tiere in Ruhe lassen." Eine volle Internalisierung aller Negationen in einem Konstrukt gelingt zum Beispiel im Konzept der Verantwortung: Der Mensch hat als am höchsten entwickeltes Lebewesen eine Prärogative (Negation der vollen Gleichberechtigung anderer Lebewesen), die ihm aber besondere Verantwortungspflichten auferlegt: Er muß in diesem Fall zurückstecken (Negation von ,Erholung'), bei essentielleren Belangen nicht (Negation der Negation). Wir werden im folgenden sehen, daß die Schlußfolgerungen der Technikfolgenabschätzung von herbizidresistenten Kulturpflanzen oft genau diese Gestalt einer Konstruktion mit internalisierten Negationen/Gegenargumenten angenommen haben. Und die Internalisierung der Negationen weist auf ein reflexives Gleichgewicht hin, weil die ursprünglichen Gegenargumente ihren Charakter als Negationen verloren haben und das Urteil nicht mehr gefährden können.

Internalisiert wird aber nur, was Bestand gehabt hat und was als Einzelbeobachtung, als Einzelargument nicht widerlegt werden konnte. Bei einer Kontroverse um eine Technik wie die der Herbizidresistenz stehen sich anfangs Hunderte von Einzelbehauptungen, die unterschiedlichen Risikoklassen zugeordnet sind, gegenüber. Viele von ihnen müssen widerlegt werden, damit ein ,internalisierungsfähiger' Bestand übrig bleibt. Der soziale Mechanismus einer Technikfolgenabschätzung sorgt auch dafür, daß dies geschieht. Durch die Analyse von Diskursleistungen wird deutlich, warum und wie derartiges geschieht.

\subsection{Diskursleistungen und Rationalisierungseffekte in der Technikfolgenabschätzung von herbizidresistenten Kulturpflanzen}

\section{(a) Setting und Verhandlungsphase}

Ich werde mich in diesem Abschnitt im wesentlichen auf Diskursleistungen bei Tatsachenbehauptungen konzentrieren, weil beim Konflikt um die Herbizidresistenz-Technik alles von diesen abhing. Dies gilt, obwohl gerade auch bei der Kontroverse um die Gentechnik unterschiedliche Wertsysteme/Kosmologien aufeinanderprallen sollen. Diese Stilisierung ist ganz definitiv falsch. Ein kurzer Blick auf die der Technikfolgenabschätzung vorausgehende Phase und auf die Verhandlungsphase der Technikfolgenabschätzung (Rekrutierung und thematische Eingrenzung) läßt, denke ich, deutlich werden, warum das so ist.

Die Herbizidresistenz-Technik ist eine Variante der chemischen Unkrautbekämpfung. Man fügt den Genomen von Kulturpflanzen Gene ein, die diese resistent werden lassen gegenüber Herbiziden mit besonders breitem Wirkungsspektrum. Diese Technik ist von 1991 bis 1993 am Wissenschaftszentrum 
Berlin (WZB) (finanziert durch das BMFT) einer umfassenden Würdigung unterzogen worden - nicht ohne Anstoß durch öffentliche Diskussionen. Die Herbizidresistenz-Technik galt als besonders problematisches Beispiel einer auch sonst abgelehnten Technik, weil sie den Einsatz von Pflanzenschutzmitteln, die immer ein toxisches Potential darstellen, begünstigen würde. In dieser vorauslaufenden öffentlichen Diskussion hatte sich eine Reihe von Bewertungsdimensionen herauskristallisiert. Sie betrafen die menschliche Gesundheit (Humantoxikologie), Gefahren für Luft, Gewässer und Boden (Ökotoxikologie), den Artenreichtum, die Stabilität des Ökosystems durch Gentransfer, Risiken für die Welternährung aufgrund mangelnder Stabilität der herbizidresistenten Sorten oder einer weiteren Reduktion der Sortenvielfalt und Monopolisierungsgefahren in der Landwirtschaft. Diese Risikodimensionen und die darin implizierten Werte waren in keiner Phase der Technikfolgenabschätzung strittig. Ausnahme ist der Wert ,Integrität des Pflanzengenoms', der im ethischen Gutachten der Technikfolgenabschätzung angesprochen wurde (Altner 1994). Er hat aber weder in der öffentlichen Diskussion noch in der Technikfolgenabschätzung eine prominente Rolle spielen können.

Soweit kann also von einem Dissens in Wertfragen überhaupt nicht gesprochen werden. Die Kontroverse um die Gentechnik in der Landwirtschaft kreist um geteilte Werte, wobei dem Wert ,menschliche Gesundheit' eine überragende Bedeutung zukommt. Der Grund für diese Konzentration auf geteilte Werte ist evident. Die Rhetorik von den unüberbrückbaren Wertdivergenzen, dem völlig argumentationstranszendenten Wertpluralismus gehört zum Allgemeingut aller auch nur oberflächlich Gebildeten. Daher erwartet man auch nicht, daß man durch Beschwörung der eigenen Werte Vertreter anderer Wertungen beeindrucken kann. Man wird dann vom Opponenten allenfalls zu hören bekommen, daß in Wertfragen nicht argumentiert werden kann und daß das Mehrheitsprinzip daher völlig zu Recht zum Zuge kommt. Es ist die einzige - soziale - Basis von Legitimität, weil es sachliche Legitimitätsgründe per definitionem nicht geben kann. Nur wenn man an geteilte Werte appellieren kann, hat man Grund, beim Gegner argumentative Bewegung zu erwarten und genau deshalb bleiben die nichtgeteilten Werte in den Kontroversen um die Gentechnik etc. weitgehend ,draußen vor'. So ist es empirisch, und so ist es, wenn man die Implikationen des Wertpluralismus in argumentationsstrategischer Hinsicht auch nur ansatzweise durchdenkt. Wenn Werte nicht strittig sind, dann verbleiben die Eintrittswahrscheinlichkeiten ihrer Verletzung, das heißt die Risiken, als Schlachtfeld; und daher waren die Diskussionen dieser Technikfolgenabschätzung über sehr, sehr weite Strecken Diskussionen über wissenschaftliche Tatsachen.

Nun sind damit keineswegs alle Wertungsfragen umschifft. Es muß festgelegt werden, über welche Tatsachen denn im einzelnen verhandelt werden soll, und in diese Konstitution des Gegenstandes der Technikfolgenabschätzung 
gehen unvermeidlich auch leitende Werte ein. Diese Festlegung der Diskussionsfelder der Technikfolgenabschätzung verlief dann auch nicht konfliktfrei. Die Herbizidresistenz-Technik ist eine Anwendung der Gentechnik im konventionellen, stark chemiegestützten Pflanzenschutz; mit diesem konventionellen Pflanzenschutz konkurriert der des ,integrierten Anbaus' (weitgehender Verzicht auf Chemie zugunsten anderer Strategien wie richtige Sortenwahl, andere Fruchtfolgen etc.) und der des Ökolandbaus (vollständiger Verzicht auf Chemie). Somit hätte man über Gentechnik allgemein, über die komparativen Vor- und Nachteile der unterschiedlichen Systeme des Pflanzenschutzes und in diesen erweiterten Kontexten dann über Risiken und Vorteile der Herbizidresistenz-Technik reden können. Diese Forderung kam auch auf: in Gestalt des Verlangens, statt der enggeführten technikinduzierten Technikfolgenabschätzung von Herbizidresistenz eine probleminduzierte Abschätzung der konkurrierenden Systeme des Pflanzenschutzes durchzuführen. Das WZB konnte diesem Verlangen nicht nachgeben, weil die Mittel nun einmal für die Abschätzung der Herbizidresistenz-Technik freigegeben waren.

Aber es war auch nicht einzusehen, warum die Risikobehauptungen, die von den Kritikern in der Öffentlichkeit verbreitet worden waren, nicht auch überprüft werden müßten. Die Kritiker hatten die Diskussionen um die Herbizidresistenz-Technik in der Öffentlichkeit nun einmal technikinduziert inszeniert. Nun hätten sie nicht ,aussteigen' können, ohne die eigene Inszenierung zu diskreditieren, und so kam es nach einigem Hin und Her schließlich zu einem working consensus dahingehend, daß eine technikinduzierte Technikfolgenabschätzung durchgeführt wurde (vgl. zu Einzelheiten Bora/Döbert 1993; Döbert 1996a). ,Gründe' allein haben in diesem Fall gewiß nicht den Ausschlag gegeben. Aber die thematische Eingrenzung eines so komplexen Gegenstandes wie "die Herbizidresistenz-Technik mit all ihren Konsequenzen“ läßt sich sowieso nicht bis ins letzte mit Argumenten abdecken. Thematischen Eingrenzungen inhäriert immer und notwendigerweise ein Moment reinen, das ist grundlosen Entscheidens, da die Systemabgrenzungen nicht wasserdicht sind (vgl. Döbert 1996a). An dem Punkt hat der soziale Mechanismus der Konsensbildung Lücken in der Sachargumentation zu überbrücken. Das ist schließlich geschehen. Und damit hat die Beschränkung auf eine technikinduzierte Technikfolgenabschätzung für die Teilnehmer der Technikfolgenabschätzung volle und nicht überbietbare Legitimität gewonnen, weil sie sie beschlossen haben. Diesen verfahrenstheoretischen Punkt hat man in der Literatur über die Technikfolgenabschätzung mehr als einmal übersehen (Saretzki 1996; Neubert 1993). ${ }^{1}$

1 Übersehen hat man aber vor allem etwas anderes. Knapp zwei Jahre vor Beginn der Technikfolgenabschätzung von herbizidresistenten Kulturpflanzen hatte die Regierung einen probleminduzierten Vergleich der konkurrierenden Landwirtschaftssysteme durchführen lassen. Diese Information hätte genügt, um die Technikinduzierung zu legitimieren. Denn eines hätte wohl jedermann eingeleuchtet: daß eine Gesellschaft derart aufwendige Unter- 
Die Kontroverse um technik- versus probleminduzierte Technikfolgenabschätzung war die einzige heikle Phase bei der Initiierung der Technikfolgenabschätzung; alle anderen möglichen Streitpunkte konnten fast geräuschlos im Modus des Verhandelns einer Einigung zugeführt werden, weil man im Prinzip durch ,Wachstum' integrieren konnte. So wurde die Forderung nach einem zusätzlichen Gutachten über die Bodenerosion umgehend aufgegriffen, mehr Diskussionszeit wurde durch Planung von ,Workshops' gewonnen und auf den Teilnehmerkreis konnte man sich auch ohne nennenswerte Störungen verständigen, da ein Konsens dahingehend herrschte, daß die Technikfolgenabschätzung sachlich und sozial repräsentativ zusammengesetzt sein sollte: Im Prinzip versuchte man, dem Gleichheitsprinzip gerecht zu werden und Fairneß zu realisieren. So gab es insgesamt dann in der Konstitutionsphase wenig Konflikte im einzelnen.

Und das war für das Gelingen der Technikfolgenabschätzung nun auch von zentraler Bedeutung. Denn sie war als ein voll partizipatives Verfahren angelegt (vgl. van den Daele/Döbert 1995), das sowohl Prozeßkontrolle wie Ergebniskontrolle realisiert. Prozeßkontrolle bezieht sich auf die Topoi, die im Verfahren aufgebracht werden dürfen; Ergebniskontrolle bezieht sich bei einer Technikfolgenabschätzung auf die Schlußfolgerungen, die am Ende an Argumenten und Gegenargumenten gezogen werden (vgl. Tyler 1990). Man kann das auch so sagen: In diesem Verfahren sollten die Teilnehmer ohne äußere Einschränkungen für die Variabilität und die anschließende Selektion zuständig sein. Das ,elitäre' Moment von Intellekt sollte also nicht - etwa wie bei Anhörungen, Erörterungsterminen etc. - auf eine dritte Instanz (etwa nach Art des TAB) verlagert werden. Der Grund für dieses Arrangement liegt darin, daß man nur so herausfinden kann, wieweit die Kraft rationaler Argumentation wirklich trägt. Wird die Selektion auf eine dritte Instanz verlagert, können die Beteiligten die Legitimationskraft von Argumentation leicht unterlaufen: Die Schlußfolgerungen sind nicht zwingend, sondern parteiisch, manipulativ etc.

nehmungen nicht im Zweijahresrhythmus würde wiederholen wollen. Daß diese Information beim Aufkommen der entsprechenden Diskussion nicht in die Arena geworfen werden konnte, hängt meines Erachtens mit einem möglichen Rekrutierungsfehler zusammen: In einer Technikfolgenabschätzung über eine Landwirtschaftstechnik hätte einer der Antragsteller aus den Agrarwissenschaften kommen müssen. Er hätte für die landwirtschaftlichen Aspekte eine besondere Verantwortung gehabt und dafür gesorgt, daß andere Technikfolgenabschätzungen von Landwirtschaftstechniken zur Kenntnis genommen werden. Das hätte die Klagen über die Technikinduzierung mit Sicherheit im Keime erstickt. 
(b) Diskursleistungen und Rationalisierungseffekte bei Tatsachenbehauptungen

Manche Schädigungen durch Technik lassen sich durch bloßen Augenschein durch jeden Laien konstatieren. Im Falle der Herbizidresistenz-Technik gilt dies für die meisten Risiken nicht. Herbizidrückstände/-umwandlungsprodukte etc. sind ,unsichtbar' und lassen sich nur durch Wissenschaft identifizieren. Insofern bleibt den Herbizidresistenz-Technik-Kritikern überhaupt nichts anderes übrig, als dem Weltbild/Naturkonzept der Wissenschaft wenigstens, den kleinen Finger zu reichen ${ }^{\text { - }}$ um das mindeste zu sagen. Nichtsdestoweniger gäbe es auch unter diesen ungünstigen argumentationsstrategischen Bedingungen noch hinreichend Spielraum für die Entfaltung anderer Naturvorstellungen. Man kann Interdependenzen und Komplexität stärker betonen und damit auf jeden Fall ,Unsicherheit' besser dramatisieren. Dementsprechend wären eventuelle Schlußfolgerungen hochgradig ungesichert und würden die eigene Position nicht unbedingt tangieren. Ich will in diesem Abschnitt versuchen zu zeigen, daß auch diese Verteidigungslinie der Kosmologietheoretiker im Fall der Herbizidresistenz-Technik allenfalls im Peripheren zu halten ist. Damit wäre dann zu vermuten: Wenn die Kontroverse um die (Herbizidresistenz-Technik als ein Fall der) Gentechnik zu einem kosmologischen Schisma hoch stilisiert wird und sich zeigt, daß weder im Evaluativen noch im Deskriptiven wirklich prinzipiell Divergentes aufeinanderprallt, dann müssen die letzten Konfliktgründe im Funktionalen, nicht im Strukturellen (kognitiv-evaluative patterns) liegen. Man hat sich qua Organisation auf die Bekämpfung der Gentechnik (Funktion) festgelegt und sucht nun nach Gründen. Anders gesagt: Es nützt gelegentlich, sich auf eine andere Kosmologie zu berufen. Ich will nicht behaupten, daß dies ,die ganze story' ist; aber ich denke, daß man demonstrieren kann, daß diese Hypothese auf jeden Fall einen erheblichen Teil der Wahrheit trifft.

Demonstrieren läßt es sich meines Erachtens dadurch, daß man die Mechanismen aufzeigt, die dafür sorgen, daß Argumente/Gegenargumente in einem Diskurs unter Selektionsdruck geraten. Nennen wir diese Mechanismen Diskursleistungen (vgl. van den Daele/Döbert 1995, Döbert 1996a). In vollem Umfang zum Tragen kommen sie, wenn im wesentlichen zwei Bedingungen erfüllt sind: (a) Die Diskursuniversalien im Sinne von Piagets „Kooperation“ (zur Übersicht Döbert 1992) oder Apel/Habermas' ,Universalpragmatik' müssen realisiert sein. (b) Bei der Rekrutierung der Technikfolgenabschätzung muß sorgfältig darauf geachtet werden, daß sämtliche relevanten Positionen/Argumente von den Teilnehmern repräsentiert werden und die Wissensbestände sich auch hinreichend überlappen. Monopolwissen macht sich sofort in argumentativen Schieflagen und Stilisierungen ${ }^{2}$ bemerkbar. Die

\footnotetext{
${ }^{2}$ Um ein Beispiel zu geben: Eine Risikoerwartung bezog sich darauf, daß es bei der Herbizidresistenz-Technik eher zur Selektion von gegen die Herbizide resistenten Unkräutern
} 
Bedingung $b$ bezieht sich auf ,Semantik ${ }^{6}$, nicht ,Pragmatik ${ }^{6}$ - was nicht überrascht, wenn man Rationalität im Sinne von ,Sachangemessenheit' faßt.

Sind beide Bedingungen erfüllt, dann kommt es bei einem Diskurs unter Anwesenden (und nur dort) sozusagen durch die reine Mechanik des Diskursablaufs und auch völlig unabhängig von den performativen Einstellungen der Beteiligten (man kann ruhig ,täuschen' wollen - man ,kommt damit nicht durch'!) zu einer Reihe von Diskursleistungen, die man als Dienstleistungen des sozialen Mechanismus Diskurs für die Sachdimension/Argumentationslage definieren kann. Die Effekte in der Sachdimension sind dann Rationalitätsgewinne. Ich lasse zunächst einige der von uns in dieser Technikfolgenabschätzung identifizierten Diskursleistungen Revue passieren, um dann an zwei Beispielen eingehender zu verdeutlichen, wie sie die Internalisierung von Negationen bzw. ein wohlerwogenes Urteil im reflexiven Gleichgewicht vorbereiten und erzwingen.

In der Wertdimension lag die entscheidende Diskursleistung, wie wir gesehen hatten, in der Fokussierung auf geteilte Werte, die auch zu Entfundamentalisierungen führt. Bei strittigen Tachsachenbehauptungen sind folgende Diskursleistungen zu nennen: Es kommt zu einer erheblichen Reduktion von Ungewißheit, da Information gepoolt wird; vorzeitige Schließungen im Sinne des Abbruchs der Argumentation vor Prüfung aller Gesichtspunkte werden durch die Opponenten konterkariert; Selektivitäten in der Informationsverarbeitung - seien es motivational bedingte Verdrängungen oder arbeitsteilig bedingte Spezialisierungen - werden durch die bloße Interaktion überwunden; durch Kooperation mit den Gleichgesinnten, aber auch durch unfreiwillige Zuarbeit der Gegner kommt es zu ,Stärke durch Vielfalt im Sinne Minskys (viele schwache Argumente stützen eine dann starke Position: Minsky 1990); Risiken werden durch Vergleiche (etwa mit konventionellen Pflanzen, konventionellen landwirtschaftlichen Praktiken), normalisiert ${ }^{t}$ und verlieren so viel von ihrem kritischen Potential (business as usual); Inkonsistenzen in der eigenen Position werden vom Gegner gnadenlos aufgespießt und nötigen zu Korrekturen; insgesamt entsteht ein Druck, monofaktorielle Erklärungen in multifaktorielle, die realen Systemen eher angemessen sind, zu überführen. Denn die relevanten Disziplinen sind präsent. Dann können auch Negationen eher internalisiert werden, weil z. B. die Wirkung eines Faktors durch die eines anderen kompensiert werden kann und man somit nicht gezwungen ist, die Relevanz des ersten in toto zu leugnen. Machen wir uns an zwei Argumentationsblöcken aus der Technikfolgenabschätzung von herbizidresistenten

käme, die dann schwer bekämpfbar wären. Bei Abwesenheit der Herbizidresistenz-TechnikKritiker hieß es dazu von seiten der Befürworter: Es gibt überhaupt keine entsprechenden Mutanten, da Mutationen in diesem Falle ,lethal mutations' sind; bei Anwesenheit der Kritiker mußte abgemildert werden zu: Es gibt die entsprechenden Mutanten, aber sie sind in ihrer Fitness erheblich geschwächt. 
Kulturpflanzen klar, wie diese Diskursleistungen im einzelnen funktionieren und welche Schlußfolgerung sie schließlich nahelegten.

Um die Informationsmassen in der Technikfolgenabschätzung zusammenzufassen, sind für die Abschlußkonferenz auch umfassende Argumentationsbäume erstellt worden, in denen sämtliche Pro- und Kontra-Argumente zu den einzelnen Risikobehauptungen enthalten waren. Sie sollten den Beteiligten den Überblick erleichtern, sie sollten aber auch das WZB vor Vorwürfen der Einseitigkeit etc. schützen. In einem der ,Bäume' ging es um die Frage, ob die Toxikologie der herbizidresistenten Pflanzen zu besonderer Besorgnis Anlaß gibt, weil neue Herbizidrückstände möglicherweise in bedenklichen Mengen in den Pflanzen angesammelt werden. In diesem ,Baum' spielte die Dramatisierung von Unsicherheit und Erkenntnislücken eine besonders herausragende Rolle. Im Prinzip hoffte man, die Technik durch bloße Ungewißheit kippen zu können. Ich drucke eine Teil des betreffenden Argumentationsblocks ab.

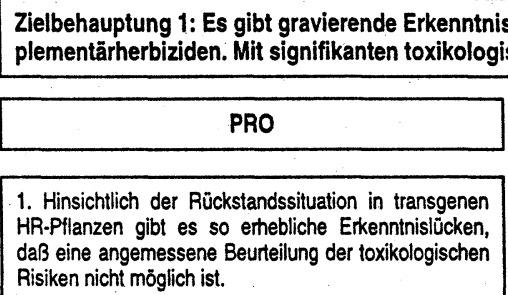
Risiken nicht möglich ist.

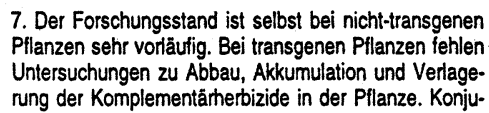
Pflanzen sehr vorläufig. Bei transgenen Pflanzen fehlen Untersuchungen zu Abbau, Akkumulation und Verlagerung der Komplementärherbizide in der Pflanze. Konjugate und Metabolite sind uneriorscht.
3. Die Tatsache, daß die Daten fưr die zulassungsrelevanten Untersuchungen nicht öffentlich zugänglich sind, rechtlertigt Zweifel an deren Wissenschattlichkeit. Eine unabhängige Überprưfung der Daten muß möglich sein.

Die beiden Herbizide, um die es in der Technikfolgenabschätzung ging, waren Basta und Round-up (zusammenfassend ,Komplementärherbizide' genannt). In toxikologischer Hinsicht sind Herbizidrückstände sowie Abbau- und Umwandlungsprodukte der Herbizide in der Pflanze relevant. Behauptung 7 läuft darauf hinaus, daß man darüber so gut wie nichts weiß. Die Kontra-Behaupt- 
ungen widersprechen dem recht gründlich und ihnen ist man schließlich gefolgt, weil die entsprechenden Erkenntnisse zum Teil in der Technikfolgenabschätzung vorgelegt wurden. Der Ablauf kann nicht überraschen, denn bei arbeitsteiliger Erkenntnisproduktion ergibt sich fast mit apriorischer Gewißheit folgende Konstellation.

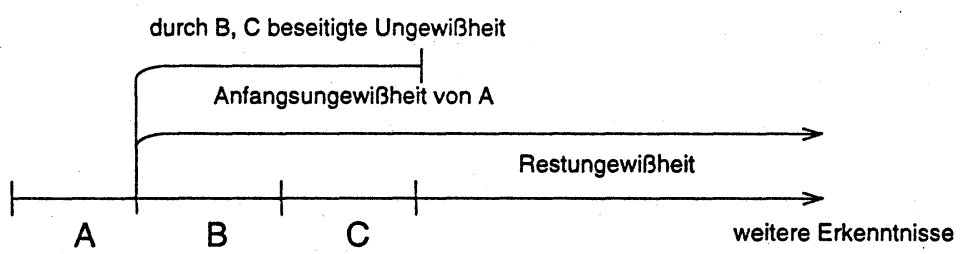

Zur Erläuterung: Seien $A, B$ und $C$ drei Spezialisten mit den ihnen zugeordneten Wissensbereichen; dann wird $A$, wenn er sich Risiken ausmalt, diese auch in die Wissensbereiche von $B$ und $C$ projizieren, da er entsprechende Gegenevidenzen nicht kennt (analog für $B$ und $C$ ). Durch die Präsenz von $B$ und $C$ im Diskurs wird ein Teil seiner Risikohypothesen automatisch zum Scheitern verurteilt sein. Denn Ungewißheit wird in erheblichem Maß abgebaut. Das kann überhaupt nicht anders sein. Nun kann $A$ immer noch im Bereich der Restungewißheit Risiken ansiedeln. Was aber finden wir im Bereich dieser Restungewißheit mit hoher Wahrscheinlichkeit? Nehmen wir an, $A, B$, $C$ repräsentierten das gesamte Spektrum des momentan verfügbaren Wissens über Herbizide und Pflanzenphysiologie. Dann liegen jenseits der Strecke $A$, $B, C$ die unbekannten Stoffklassen und die mit verfügbaren Geräten nicht meßbaren, verschwindend geringen Stoffmengen. Die können zur ,Konstruktion' spekulativer Risiken herangezogen werden und das geschieht auch. Aber es geschieht eben erst, nachdem die Risiken in den Intervallen $B$ und $C$ benutzt worden sind, und das spricht doch sehr dafür, daß der Rekurs auf die Restrisiken sich nicht einem strukturell anderen Weltbild mit höherer Risikoempfindlichkeit verdankt, sondern funktional bestimmt ist: Andere Argumente gibt es nicht mehr. Und nicht nur das. Diese Argumente sind unspezifisch und lassen sich nicht gezielt gegen einzelne Pflanzensorten benutzen. Sie unterliegen der Normalisierung durch Vergleich. Unbekannte Stoffe in nicht meßbaren Mengen kennen wir bei keiner Pflanze: „Der Forschungsstand ist selbst bei nicht-transgenen Pflanzen sehr vorläufig" heißt es in Argumentbox 7 und das relativiert die argumentative Stärke von Restungewißheit doch sehr.

Der Diskurs läßt also insgesamt deutlich werden, daß die Dramatisierung von Ungewißheit ganz einfach auch - bei Arbeitsteilung ganz unvermeidliche Kenntnislücken anzeigt. Und er zeigt auch, daß verbleibende Restungewißheiten nicht den Status generalisierter ,kosmologischer' Konzepte haben können, 
weil derartige Restungewißheiten ubiquitar sind und daher in vielen Bereichen übergangen werden müssen. Nachweisbar sind also Kenntnislücken und einseitige Fokussierungen auf bestimmte Restrisiken. Sie reichen aus, um die Haltung zur Gentechnik zu ,erklären' und daher gibt es eben keinen nachweisbaren Anlaß zu kosmologischen Spekulationen.

Nun ein Ausschnitt aus einem Argumentationsbaum, in dem es um die Gefahr ging, daß bei der Herbizidresistenz-Technik mehr Herbizide ausgebracht werden würden, weil die resistenten Kulturpflanzen durch die Herbizide in keinerlei Weise beeinträchtigt werden könnten (,Mengenbaum $\left.{ }^{6}\right)$. Der Ausschnitt ist immer noch so umfangreich, daß ich ihn in den Anhang verbanne und hier eine stilisierte Zusammenfassung gebe, die einige Diskursleistungen sichtbar werden läßt. Die Diskursleistung der ,Stärke durch Vielfalt ${ }^{t}$ verdeutlicht man sich am besten, wenn man die Argumentationsbatterie jeweils einer Seite, Pro oder Kontra, jeweils für sich, ohne auf die Gegenseite zu schauen, liest. Es ergibt sich ein starker ,Sog', der sozial dadurch zustande kommt, daß mehrere Spezialisten ihre Kenntnisse in die gleiche Waagschale werfen. Die Herbizidresistenz-Technik-Gegner argumentieren in diesem Falle in etwa wie folgt: Die Bauern können bei der Herbizidresistenz-Technik große Herbizidmengen verwenden, weil die Kulturpflanzen nicht geschädigt werden. Und sie werden das auch tun, weil es inkompetente Bauern gibt; weil sie bei der Unkrautbekämpfung auf Sicherheit bedacht sind; weil sie routinemäßig, ohne Rücksicht auf Bedarf, spritzen; weil sie mit dem Kauf des Saatguts die Herbizide bezahlt haben; weil die Herbizidresistenz-Technik zum Dauereinsatz der gleichen Herbizide verführt und weil Daueranbau schließlich zur Selektion resistenter Unkrautpopulationen führt, die dann mit erhöhten Herbizidmengen bekämpft werden müssen. Das ist ,Stärke durch Vielfalt', bei der ohne weiteres ein Glied aus der Kette gebrochen werden kann, ohne daß das Gewicht des Ganzen gefährdet wäre.

Wenn man von dem auf einem Mißverständnis beruhenden Argument absieht, daß der Bauer das Herbizid mit dem Saatgut bezahlt, zeichnen sich sämtliche verbleibenden Argumente durch eine entscheidende Selektivität aus: Sie abstrahieren vollständig von der ökonomischen Seite der Technik, nämlich den Herbizidkosten. Diese Selektivität wird durch die Gegenseite aufgehoben: Es „wird heute aus Kostengründen meist unterdosiert" (Anhang, Argument 2). Das von niemandem bestrittene Argument, bei dem Herbizid Atrazin seien große Mengen ausgebracht worden, ließ sich als irrelevanter Sonderfall verdrängen/beiseite schieben. Atrazin ist inzwischen verboten und war unvergleichlich billig.

Damit stand die Technikfolgenabschätzung am Punkt einer vorzeitigen Schließung der Diskussion: Man wollte dem Risiko der Überdosierung auch die geringste Plausibilität absprechen. Diese vorzeitige Schließung ist dann aber aufgehoben worden durch die Aufdeckung einer Inkonsistenz, die nun 
wiederum die technische Seite des Herbizideinsatzes betrifft. Die Technikfolgenabschätzung hatte der Resistenzproblematik (Selektion resistenter Unkräuter) einen eigenen Argumentationsbaum gewidmet, weil es sich um eine reale Gefahr handelt. Resistente Unkräuter müssen aber mit hohen Dosierungen bekämpft werden. Das Problem ließ sich also nicht so einfach beiseite schieben. Nun reagiert die Gegenseite mit einer Differenzierung im Sinne Piagets: Es gibt in der Unkrautbekämpfung ein Mißmanagement mit entsprechenden Fehlentwicklungen („Dumme gibt es überall“) und das, was man ,gute landwirtschaftliche Praxis' nennt. Das Mißmanagement wird dann aber normalisiert: es ist nicht spezifisch für die Herbizidresistenz-Technik (Box 19). Zudem kann man den Daueranbau von Kulturen, der am ehesten zu Resistenzen führt, verbieten (Box 11).

Regulierung ist also die dritte Variable, die neben die technischen und ökonomischen Aspekte gestellt werden muß, will man zu einer angemessenen multifaktoriellen Beschreibung des Systems Landwirtschaft gelangen. Auf eine solche multifaktorielle Beschreibung lief die Technikfolgenabschätzungsdiskussion letztendlich zu. Denn der Relevanz dieser Variablen hat niemand grundsätzlich widersprechen können.

Ich gebe die Schlußfolgerung, die aus der kontroversen Diskussion gezogen wurde, in komprimierter Form wieder. Dabei sind die Internalisierungen der Negationen, die diese Kontroverse bestimmt haben, hervorgehoben:

Der Herbizideinsatz in der Landwirtschaft wird von technologischen Möglichkeiten/Restriktionen und Kostenerwägungen bestimmt, wobei der Kostenfaktor einen Generaltrend zur Verringerung des Herbizideinsatzes bedingt. Abgesehen von einer unspezifischen Fehlerrate beim Herbizidmanagment $(\mathrm{Ne}-$ gation 1) ist mit erhöhten Dosierungen zu rechnen, wenn Kostenerwägungen wie bei Atrazin eher irrelevant sind (Negation 2), oder wenn ohne eine Erhöhung des Herbizidaustrags größere Schäden an der Ernte drohen - Stichwort ,resistente Unkräuter' (Negation 3). Mit Negation 2 und 3 ist bei Glufosinat und Glyphosat nicht zu rechnen, speziell dann nicht, wenn man die ohnehin geringe Resistenzgefahr beider Herbizide noch durch ein Verbot des Daueranbaus (Negation 4) absichert.

Diese Schlußfolgerung ist, so meine Behauptung, bombenfest, weil sie alle berechtigten Argumente der Herbizidresistenz-Technik-Kritiker in sich aufnimmt und lediglich Variablen koordiniert, die generell in ihrer Relevanz unstrittig waren. Sie überwindet die Einseitigkeit einer rein technischen oder rein ökonomischen Betrachtung, weil der Diskurs schließlich die umfassende Sicht erzwungen hat, die die Teilnehmer selbst auch gefordert hatten. Und sie bewahrt auch alle Negationen, die als unbestreitbare Tatsachen nicht zurückgewiesen werden konnten.

So hat der Diskurs also das Niveau von Rationalität erzeugt, das oben postuliert worden ist. Dabei zeigt der Gang der Diskussion wieder und wie- 
der, daß die Ausgangspositionen der Opponenten in erheblichem Umfang auf Selektivitäten und Einseitigkeiten - von berücksichtigten Variablen, von vergleichbaren Gefahren etc. - aufbauen. Der Diskurs zwingt schließlich alle Variablen und Gesichtspunkte zusammen. Daß dabei eine umfassende Beschreibung auf der Basis unstrittiger Variablen zustandekommen konnte, zeigt auch an, daß sich in der Kontroverse um die Herbizidresistenz-Technik nicht inkompatible Kosmologien gegenüberstehen. Man sprach weitgehend eine Sprache und das war die Sprache der Wissenschaft.

Somit besteht wenig Grund zur Annahme, daß die Wissenschaft nur eine Verkörperung von vier gleichberechtigten ,Rationalitäten' (Douglas' Kosmologien) darstellt. Sie hat sich als die überlegene eine Rationalität erwiesen, die herzustellen allerdings ein mühsames Unterfangen ist. Das hypothetische Risiko eines erhöhten Herbizidaustrags war argumentativ damit weitgehend erledigt. Inzwischen sind herbizidresistente Pflanzen (Soja) auf den amerikanischen Markt gebracht worden und (vorläufig) erfolgreich, weil die Landwirte bei diesen Kulturen Herbizide einsparen können. Objektiv sind die Diagnosen der Technikfolgenabschätzung in dem Punkt also bestätigt worden. Was heißt das für die Rationalität der Handlung ,Bekämpfung der HR-Technik'? Es heißt auf jeden Fall, daß sie, soweit sie mit den in der TA unterbreiteten Gründen gerechtfertigt wird, Rationalität im stärksten Sinne nicht verkörpern kann. Jedenfalls ergibt sich dies für einen Beobachter der Argumentationslage.

Das sagt nun über die Akzeptabilität der Schlußfolgerungen für die Herbizidresistenz-Technik-Kritiker wenig. Denn diese sind bekanntlich vor der endgültigen Modifikation und Verabschiedung der Schlußfolgerungen dieser Technikfolgenabschätzung aus dem Verfahren ausgestiegen. Beweist das nicht, daß sie im Rahmen ihres Weltbildes die Schlußfolgerungen einfach nicht als ,richtig', ,schlüssig', ,zwingend' erkennen konnten? So hören wir den Neokonstruktivisten und Kosmologen ohne Zweifel wieder unken! Bei politisierten Kontroversen kann man jedoch nicht von argumentativer Schlüssigkeit auf Akzeptanz schließen: Etwas kann gerade deshalb inakzeptabel sein, weil es schlüssig ist und damit selektiv/elitär im eingangs bezeichnetem Sinne. Ehe wir diesen Punkt abschließend kurz erörtern, sei jedoch auf ein indirektes Indiz für die Schlüssigkeit der Konklusionen der Technikfolgenabschätzung hingewiesen.

Ich hatte oben hervorgehoben, daß für das Wirksamwerden von Diskursleistungen die richtige Rekrutierung von ausschlaggebender Bedeutung ist. Fehlende Kompetenz in einem Teilbereich erzeugt sofort Störungen. Und einen Rekrutierungsfehler wird man der Technikfolgenabschätzung vielleicht bescheinigen müssen (siehe oben, Fußnote 1): Man hatte zwar genügend landwirtschaftliche Experten und Expertinnen rekrutiert, aber unter den Antragstellern (W. Daele, A. Pühler, H. Sukopp) fehlte jemand aus diesem Bereich. Die Gutachten und Diskussionen der Technikfolgenabschätzung mußten am Ende zu vorläufigen Schlußfolgerungen verdichtet werden. Dies hat dem dafür 
zuständigen WZB- Team einige Mühen bereitet. Ein zuständiger Antragsteller/eine Antragstellerin wäre sicherlich eine große Hilfe gewesen. So ist es faktisch dazu gekommen, daß die Schlußfolgerungen für den landwirtschaftlichen Teil der Technikfolgenabschätzung vor Beginn der Abschlußkonferenz nicht ganz fertig und ausgereift waren. Da gab es scheinbar argumentative Grauzonen, in denen manche Risikothese überleben konnte. Und diese Grauzonen wurden denn auch genutzt. Die Herbizidresistenz-Technik-Kritiker haben ihren Ausstieg in einem Flugblatt begründet und sich auch noch einmal in einer abschließenden Befragung (Gill 1993) zum Thema geäußert. Recht unzweideutig geht aus beiden Quellen hervor, daß man sich vor allem in seinen mit dem Herbizideinsatz verbundenen Risikothesen bestätigt sah. Die gentechnikbezogenen Risikohypothesen tauchen kaum auf. Die entsprechenden Schlußfolgerungen waren bei Beginn der Abschlußkonferenz auch weitgehend abgedichtet. Fazit: Ganz hat man sich der Kraft von Argumentation dann doch nicht entziehen können.

\section{(c) Schlüssigkeit und Akzeptanz}

Den auf Geltungsfragen spezialisierten Philosophen genügt es zu Recht, bei einer gegebenen Argumentationslage die ,überlegene' Konklusion ausfindig zu machen und zu rekonstruieren. Von daher ergibt sich rationales Handeln dann von selbst. Sozialwissenschaftlich betrachtet verhält es sich anders: Wahrheit ist auch eine knappe Ressource oder ein Handlungshindernis. Dies wird unmittelbar deutlich, wenn man die rationalistisch verkürzte Sprechakttheorie von Apel/Habermas um den unterschlagenen perlokutionären Aspekt ergänzt (vgl. Searle 1969). Jede sprachliche Äußerung läßt sich einmal in sich, isoliert von weiteren Handlungskontexten und Effekten des Sprechens analysieren. Das ist der illokutionäre Gesichtspunkt, bei dem wir auch auf unvermeidliche Geltungsansprüche stoßen (Behaupten, Versprechen, Kritisieren, Warnen etc.). Jede sprachliche Handlung läßt sich aber auch von den intendierten Effekten auf den Adressaten her charakterisieren. Das ist die perlokutionäre Beschreibung. Die in sie eingehenden Intentionen der Sprecher stehen in einem kontingentem Verhältnis zu Wahrheit/Geltungsansprüchen. Wenn ich mein Auto zu einem guten Preis verkaufen will, kann die Wahrheit mir sehr schaden - oder auch nicht. Sprechen vollzieht sich in der Regel en passant, im Vollzug weit über den einzelnen Sprechakt hinausgreifender Handlungen, z. B. der Handlung ,Kampf gegen die Gentechnik'. Und wenn die Intentionen dieser übergeordneten Handlungen mit ,Geltung' inkompatibel sind, dann muß als offen gelten, ob die Akteure sich für ,Wahrheit' oder ,Täuschung' entscheiden. Die Informationsökonomie hat sich als Disziplin etablieren können, weil es diese endemischen Konflikte zwischen Illokution und Perlokution gibt (Keck 1993). 
Schaut man sich die typischen Motivlagen von Beteiligten an den Umweltkonflikten an, dann stößt man genau auf die vor diesem Hintergrund erwartbaren Ambivalenzen. Technikfolgenabschätzungen haben eine offizielle Hauptfunktion (sozialer Frieden, Konsens auf der Basis eines begründeten Urteils); für die beteiligten Akteure können sie aber auch partikulare Nebenfunktionen erfüllen, die der Akzeptanz eines begründeten Urteils über die Technik entgegenstehen. Folgende Tabelle enthält in etwa die typische Konstellation für die Hauptakteure.

Haupt- und Nebenfunktionen bei Technikfolgenabschätzungen

\begin{tabular}{|l|c|l|}
\hline \multicolumn{1}{|c|}{ Akteure/Systeme } & $\begin{array}{c}\text { Offizielle Hauptfunktion: } \\
\text { Sozialer Frieden durch be- } \\
\text { gründetes Urteil }\end{array}$ & $\begin{array}{c}\text { Partikulare Nebenfunk- } \\
\text { tionen }\end{array}$ \\
\hline $\begin{array}{l}\text { Offentlichkeit }{ }_{n} \text { Gesamt- } \\
\text { gesellschaft }\end{array}$ & + & $\begin{array}{l}\text { Durchsetzung der Tech- } \\
\text { nikTechnikakzeptanz; public } \\
\text { relations }\end{array}$ \\
\hline Technikbetreiber & $(+)$ & $\begin{array}{l}\text { Entpolitisierung, Legitimation } \\
\text { der bisherigen Entscheidungs- } \\
\text { praxis }\end{array}$ \\
\hline Behörden & $(+)$ & $\begin{array}{l}\text { Politisierung, Mobilisierung, } \\
\text { Verzogerungstaktiken, Identi- } \\
\text { tätserhaltung }\end{array}$ \\
\hline Umweltbewegungen & $(+)$ & \\
\hline
\end{tabular}

Nur die Öffentlichkeit/Gesamtgesellschaft hat ein ungetrübtes Verhältnis zur offiziellen Hauptfunktion einer Technikfolgenabschätzung, und deshalb hat hier ein begründetes Urteil/Wahrheit eine gute Chance. Das sagt das Pluszeichen in der entsprechenden Spalte. Bei allen anderen Akteuren ist das Pluszeichen eingeklammert, weil ,Wahrheit' unter dem Vorbehalt der partikularen Nebenfunktionen steht. Die Chancen für die Akzeptanz eines begründeten Urteils liegen damit bei 50:50. Denn Akzeptanz erzeugt Handlungskosten: Man kann schlechter ,mobilisieren', die Organisationsidentität ist bedroht etc. Speziell bei Bedrohung der Organisationsidentität wird man mit Akzeptanz/Konsens nicht mehr rechnen dürfen. „Die Umwelt ist überhaupt nicht bedroht", ist ein deskriptiver Satz, der die Umweltbewegung/Umweltorganisationen in ihrer Existenz bedroht, ja sie mit einem Schlag vernichten würde - wenn er denn ,wahr' wäre. Solange es nur einen Hauch eines begründeten Zweifels an seiner Wahrheit gibt, werden die Umweltorganisationen ihn nicht konsentieren. Und solange die Antigentechnikbewegung auf Fundamentalablehnung der Technik beharrt, wird auch eine positive oder neutrale Gesamtbewertung einer einzelnen Anwendung der Gentechnik - zum Beispiel HerbizidresistenzTechnik in der Landwirtschaft - nicht konsensfähig sein. Denn ein solches 
Ergebnis bedroht die Organisationsidentität - das sind die Handlungskosten. Und diese Handlungskosten sollen sich die betroffenen Akteure im Rahmen partizipativer Arrangements auch noch selbst auferlegen und das dann vor ihren Herkunftsorganisationen vertreten - das sind die Partizipationskosten. Ich schließe den Argumentationsgang mit einem Selbstzitat:

\begin{abstract}
„Bei umfassender Partizipation soll der Delinquent die Indizien gegen sich selbst zusammentragen, sich im ungünstigsten Fall zum Tode verurteilen und sich dann auch noch selbst aufhängen. Das wird er in der Regel nicht tun." (Döbert 1996, 333f.)
\end{abstract}

Weil dies so ist, kann aus ausbleibender Zustimmung zu Schlußfolgerungen aber auch nicht auf deren mangelnde Validität geschlossen werden. Die Rationalitätsfrage ist gesondert zu prüfen, indem man sich in die einzelnen Argumentationsblöcke einarbeitet und die Schlußfolgerungen überprüft. Ich habe in diesem Artikel versucht zu zeigen, daß Ausgangspositionen der Beteiligten ihre Plausibilität überwiegend einer unvermeidlichen Selektivität der Informationsverarbeitung verdanken, die der soziale Mechanismus Diskurs überwindet. Am Ende werden Deutungen der Herbizidresistenz-Technik nicht an einzelnen Daten, Argumenten geprüft, sondern an (fast) allen. Jede Teilinformation wirkt als zusätzlicher Filter, durch den die Gesamteinschätzung der Technik hindurch muß. Dabei werden die Spielräume naturgemäß immer enger und es ,überleben' eben nicht alle Deutungen als gleichberechtigte Rationalitäten oder Kosmologien. Damit sind wir wieder am Ausgangspunkt dieses Artikels: Erkenntnis ist immanent elitär! Sie richtet sich gegen einige Akteure, die eben die Last des Irrtums tragen müssen. Und diese Last wird um so schwerer, je mehr sie durch die Last der Verletzung partikularer Nebenfunktionen ergänzt wird. Damit wird dann aber auch ein umfassender Konsens unwahrscheinlicher.

Die offizielle Hauptfunktion von partizipativen Arrangements ist damit gewiß nur noch mit starken Einschränkungen zu erfüllen. Sie ist aber keineswegs in toto gefährdet. Denn erstens unterliegen derartige Verfahren auch der Beobachtung durch die Öffentlichkeit und anderer Instanzen. Diese können sich zunächst einmal der Tatsache vergewissern, daß eben doch nicht alle Ankerpunkte für rationales Entscheiden in einem relativistischen Brei ,gleich rationaler Kosmologien' versinken müssen. Und wenn dann sorgfältig alle Risikobehauptungen geprüft sind, wenn eine Verletzung elementarer moralischer Regeln (Schädigungen, z. B. der Gesundheit, unterlassen!) nach bestem Wissen und Gewissen ausgeschlossen werden kann, dann hat das Mehrheitsprinzip die uneingeschränkte Legitimität auf seiner Seite und das läßt sich dann eben zeigen. Und zweitens macht es auch für die argumentativ unterlegene Partei einen Unterschied, ob ihre Argumente innerhalb des offiziellen Institutionengefüges überhaupt kein Gehör finden oder sorgfältig geprüft werden. Dies ist der Unterschied zwischen denen, die inkludiert werden und denen, für die to- 
tale Exklusion gilt. Und nicht zu vergessen: Auf Dauer agiert niemand in der Öffentlichkeit mit Argumenten wider besseres Wissen erfolgreich. Langfristig haben partizipative Arrangements im demokratischen Institutionengefüge daher trotz Ausbleibens umfassender Konsense durchaus eine ergänzende Funktion.

\section{Anhang}

Zielbehauptung 2: Die HR-Technik wird zu einer Steigerung des Herbizideinsatzes führen. Komplementärherbizide leisten dem Herbizidmißbrauch Vorschub, weil nicht die Gefahr besteht, daß ihr Einsatz die

Kulturen schädigt.

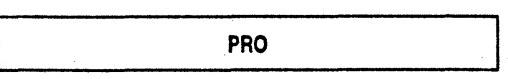

1. Da bei Komplementärherbiziden die möglichen Autwandmengen nicht schon durch die Empfindlichkeit der Kulturpflanze begrenzt werden, ist mit einem optimalen Einsatz nicht zu rechnen. "Mißbrăuche" und "Überdosierungen" sind zu enwarten.

3. Bei Atrazin wurden sehr hohe Autwandmengen eingesetzt.

5. Die Bauern spritzen lieber einmal zuviel als einmal zu wenig, um bei der Unkrautbekämpfung auf Nummer sicher zu gehen (Versicherungsprinzip).

7. Es gibt unterschiedlich kompetente Landwirte. Ne benenwerbslandwitte verwenden aus Unwissen oft zu hohe Dosierungen.

8. Die Tatsache, daß sich bei einer Reihe von Herbiziden resistente Unkräuter entwickelt haben, beweist, daß das Herbizidmanagement mangelhaft ist, also Herbizidmißbrauch vorkommt.

10. Die HR-Technik vertührt besonders zu Routinespritzplänen, weil Komplementäherbizide technisch einfach einsetzbar sind und Schäden für die Kultur nicht befürchtet werden müssen.

12. Die HR-Technik wird zu Routinespritzungen unabhängig von Schadenschwellen führen, wenn Saatgut und Komplementärherbizid , im Paket" gekautt werden, weil der Landwirt die Unkrautbekämpfung dann ja ohnehin schon bezahlt hat.

KONTRA

2. Der Begriff "Überdosierung“ ist unklar. Im Verhältnis zur zugelassenen Menge wird heute aus Kostengründen meist "unterdosiert". Die zugelassenen Mengen werden nur zu etwa $80 \%$ ausgeschöptt. Hohe Autwandmengen sind der Ausnahmetall.

4. Die hohen Dosierungen waren zugelassen. Atrazin war sehr billig und muB als Sondertall gelten.

6. Das ist „blaue Theorie; in der Praxis gilt das nicht.

. Es gibt beim Herbizideinsatz durchaus Verstöße gegen die Regeln der guten landwirtschaftlichen Praxis. Solche Vollzugsdefizite sind aber nicht HR-spezifisch.

11. Gegen Routinespritzungen sprechen. Kostengründe. Komplementämerbizide begünstigen, gerade weil sie die Kulturen nicht schädigen, die gezielte Unkrautbekămpfung je nach Bedart, also unter Berücksichtigung quantitativer Schadensschwellen.

13. Paketverkäufe" sind ökonomisch unsinnig und nicht zu erwarten, da sie die Markteinführung der HR-Technik behindern (vgl. dazu Heft 11: „Genetische Verarmung"). Ein Landwirt, der HR-Saatgut kautt, enwirbt lediglich eine Option für den Einsatz des Komplementärherbizids. Er kann Kosten sparen, wenn er die Option nicht wahrnimmt und bei fehlendem Unkrautdruck aut den Herbizideinsatz verzichtet. 


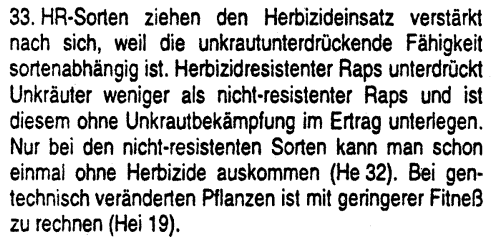

14. Die HR-Technik macht den Daueranbau herizidintensiver Kulturen, einseitiger Fruchtiolgen und Industriepflanzen mit hohem Herbizideinsatz möglich. Dadurch wird der Herbizidverbrauch steigen.

16. Aus ökonomischen Gründen kann es u. U. doch zum Daueranbau kommen.

18. Die HR-Technik könnte das Problem resistenter Unkräuter verschärfen. Als Mechanismen der Resistenzbildung kommen neben der Selektion von resistenten Mutanten auch horizontaler und vertikaler Gentrans. fer in Betracht. Die Resistenzproblematik stellt sich vor allem bei Daueranwendung der Komplementärherbizide. Sie wird dazu führen, daß die Landwirte erheblich höhere Aufwandmengen einsetzen.
34. Glufosinat (Basta) wird von resistenten Pflanzen in hohen Dosen "symptomlos" vertragen. Bei Glyphosat (Round-up) gibt es allerdings noch Schwierigkeiten (BÖ 25/37). Sorten mit verminderter Fitneß werden sich nicht durchsetzen.

15. Ausschlaggebend für Daueranbau und einseitige Fruchtfolgen sind die ökonomischen Rahmenbedingungen, nicht Optionen der Unkrautbekämpfung (vgl. Heft 11: "Genetische Verarmung"). Solche Anbauformen verbieten sich aus vielen anbautechnischen Gründen.

17. Der Einsatz der HR-Technik im Daueranbau sollte durch Regulierung ausgeschlossen werden. (Siehe nSzenarien" des TA-Veriahrens).

19. Als Mechanismus der Bildung resistenter Unkräuter ist nur die Selektion von resistenten Mutanten praktisch

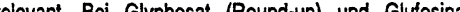
(Bant. Bei Glyphosat (Roundup) und Glusosinat (Basta) sind Resistenzentwicklungen unwahrscheinlicher als bei anderen Herbiziden (vgl. Heft 12, Abschnitt: „Entwicklung resistenter Unkräuter").

\section{Bibliographie}

Altner, G. (1994), Ethische Aspekte der gentechnischen Veränderung von Pflanzen. Gutachten erstellt im Auftrag des WZB, in: W. van den Daele/A. Pühler/H. Sukopp (Hrsg.), Verfahren zur Technikfolgenabschätzung des Anbaus von Kulturpflanzen mit gentechnisch erzeugter Herbizidresistenz, Heft 17, Berlin: WZB, discussion paper FS II 94-317

Beck, U. (1986), Risikogesellschaft: Auf dem Weg in eine andere Moderne, Frankfurt am Main

Bora, A./R. Döbert (1993), Konkurrierende Rationalitäten: Politischer und technisch-wissenschaftlicher Diskurs im Rahmen einer Technikfolgenabschätzung, in: Soziale Welt 44, 75-97

van den Daele, W. (1997), Risikodiskussionen am „Runden Tisch“, in: R. Martinsen (Hrsg.), Politik und Biotechnologie, Baden-Baden, 281-301

- /R. Döbert (1995), Veränderungen der äußeren Natur - Partizipative Technikfolgenabschätzung (TA), in: Ch. Hubig/G. Ropohl (Hrsg.), Funkkolleg Technik, Studienbrief 4, Tübingen: DIFF, 4-35

Döbert, R. (1989), Max Webers Handlungstheorie und die Ebenen des Rationalitätskomplexes, in: J. Weiß (Hrsg.), Max Weber heute, Frankfurt am Main, 210-249

- (1992), Die Entwicklung und Überwindung von ,Universalpragmatik' bei Piaget, in: Zeitschrift für Soziologie 21, 96-109 
- (1996a), Verhandeln - Entscheiden - Argumentieren in welchem Kontext?, in: V. v. Prittwitz (Hrsg.), Verhandeln und Argumentieren, Opladen, 169-182

- (1996b), §218 vor dem Bundesverfassungsgericht. Verfahrenstheoretische Überlegungen zur sozialen Integration, in: W. van den Daele/F. Neidhardt (Hrsg.), Kommunikation und Entscheidung. Politische Funktionen öffentlicher Meinungsbildung und diskursiver Verfahren. WZB Jahrbuch 1996, Berlin, 327-367

-/Th. Gericke (1997), Grundlagen der Verrechtlichung von Natur: Die Entstehung und Verankerung von Umweltnormen im umgangssprachlichen Bewußtsein, in: E.-J. Lampe (Hrsg.), Entstehung von Rechtsnormen, Frankfurt am Main, im Erscheinen

Douglas, M. (1992), Risk and Blame. Essay in Cultural Theory, London

Fischer, F. (1990), Technocracy and the Politics of Expertise, Newbury Park

Gill, B. (1993), Partizipative TA aus der Sicht von Umweltgruppen - Probleme, Ressourcen, Perspektiven, Berlin: WZB, unveröffentlichtes Manuskript

Habermas, J. (1968), Technik und Wissenschaft als Ideologie, Frankfurt am Main

Hawkesworth, M. E. (1988), Theoretical Issues in Policy Analysis, Albany

Inglehart, R. (1989), Kultureller Umbruch, Frankfurt am Main

Japp, K./W. Krohn (1996), Soziale Systeme und ihre ökologischen Selbstbeschreibungen, in: Zeitschrift für Soziologie 25, 207-222

Jungermann, H./P. Slovic (1993), Charakteristika individueller Risikowahrnehmung, in: W. Krohn/G. Krücken (Hrsg.), Riskante Techologien: Reflexion und Regulation, Frankfurt am Main, 79-100

Keck, O. (1993), Information, Macht und gesellschaftliche Rationalität, BadenBaden

Lasch, C. (1982), Das Zeitalter des Narzissmus, München

Luhmann, N. (1984), Soziale Systeme, Frankfurt

- (1986), Ökologische Kommunikation, Opladen

Minsky, M. (1990), Metropolis, Stuttgart

Naess, A. (1989), Ecology, Community and Lifestyle, Cambridge

Neubert, S. (1993), Gesellschaftlicher Dialog über umweltpolitische Streitfragen, Basel, Social Strategies

Piaget, J. (1975), Biologische Anpassung und Psychologie der Intelligenz, Stuttgart

Perrow, C. (1987), Normale Katastrophen, Frankfurt am Main

Rawls, J. (1971), A Theory of Justice, London et al.

Rucht, D. (1996), Modernisierung und neue soziale Bewegungen, Frankfurt am Main

Saretzki, Th. (1996), Verhandelte Diskurse? in: V. v. Prittwitz (Hrsg.), Verhandeln und Argumentieren, Opladen, 135-168

Sartori, G. (1992), Demokratietheorie, Darmstadt

Searle, J. R. (1969), Speech Acts, London

Simmel, G. (1900), Philosophie des Geldes, Frankfurt am Main

Teubner, G. (1989), Recht als autopietisches System, Frankfurt am Main

Thompson, M./R. Ellis/A. Wildavsky (1990), Cultural Theory, Boulder

Tyler, T. R. (1990), Why People Obey the Law, New Haven

Wagner, P. (1995), Soziologie der Moderne, Frankfurt am Main

Weber, B. (1994), Evolutionsbiologische Argumente in der Risikodiskussion am Beispiel der transgenen herbizidresistenten Pflanzen. Gutachten erstellt im Auftrag 
des WZB., in: W. van den Daele/A. Pühler/H. Sukopp (Hrsg.), Verfahren zur Technikfolgenabschätzung des Anbaus von Kulturpflanzen mit gentechnisch erzeugter Herbizidresistenz, Heft 5. Berlin: WZB, discussion paper FS II 94-305

Weidner, H. (1996), Freiwillige Kooperationen und alternative Konfliktregelungsverfahren in der Umweltpolitik. Auf dem Weg zum ökologisch erweiterten Neokorporatismus, in: W. van den Daele/F. Neidhardt (Hrsg.), Kommunikation und Entscheidung. Politische Funktionen öffentlicher Meinungsbildung und diskursiver Verfahren. WZB Jahrbuch 1996, Berlin, 195-231 